\title{
Recursos tecnológicos: estratégia de promoção do autocuidado, atividades e participação para pessoas com doença de Parkinson
}

\author{
Technological resources: strategies of promotion of \\ self-care, activities and the participation of people \\ with Parkinson's disease
}

Almeida, M. H. M. ${ }^{1}$, Castiglioni, M. C. ${ }^{1}$

ALMEIDA, M. H. M.; CASTIGLIONI, M. C. Recursos tecnológicos: estratégia de promoção do autocuidado, atividades e participação para pessoas com doença de Parkinson. Rev. Ter. Ocup. Univ. São Paulo, v.18, n. 3, p. 152-157, set./dez. 2007.

RESUMO: O envelhecimento populacional é considerado um fenômeno mundial, e a suscetibilidade para enfermidades crônicas e incapacidades decorre em grande parte do envelhecimento. Dentre os males crônicos potencialmente incapacitantes, está a doença de Parkinson (DP), que compromete progressivamente os movimentos, além de poder provocar manifestações não motoras como distúrbios de memória e depressão. Entre as medidas para a prevenção de enfermidades da idade avançada e a promoção da saúde, destaca-se o desenvolvimento de práticas de autocuidado. A adoção de recursos tecnológicos (RT) se inclui nessas práticas e inscreve-se como possível estratégia a ser empregada pelo terapeuta ocupacional para a melhora da funcionalidade. Nesse contexto, sua utilização está relacionada ao exercício de funções específicas e à prática de atividades contextualizadas. A relação entre usuário e RT tem sido comumente analisada com base nas dificuldades funcionais e na identificação de dispositivos potencialmente adequados para seu enfrentamento. No entanto, há outros referenciais a contemplar como o sentido que o usuário atribui ao dispositivo, o acesso a ele e sua permanência na vida cotidiana. O terapeuta ocupacional analisa a complexa relação entre usuário - inserido num contexto físico e social -, e dispositivo. No que tange à relação entre usuário com DP e dispositivo, pode-se afirmar que o contexto desse usuário é freqüentemente adverso - ele normalmente vive situações de dupla perda de autonomia e exclusão, tanto por ser idoso quanto por ter a DP. Cabe ao terapeuta ocupacional conceber dispositivos que exerçam função facilitadora de relacionamentos, favorecendo o resgate de sua autonomia e inclusão.

PALAVRAS-CHAVE: Doença de Parkinson. Promoção da Saúde. Autocuidado. Terapia Ocupacional.

\footnotetext{
1. Profs. Dras. Docentes do Curso de Terapia Ocupacional da Faculdade de Medicina da Universidade de São Paulo - FMUSP. Endereço para correspondência: Departamento de Fisioterapia, Fonoaudiologia e Terapia Ocupacional da FMUSP. Rua Cipotânea, 51. Cidade Universitária. São Paulo, SP. CEP: 05360-160.
} 


\section{INTRODUÇÃO}

$\mathrm{O}$ envelhecimento populacional é considerado um fenômeno mundial. Segundo o Censo de 2000 (FUNDAÇÃO IBGE, 2000), a população de idosos no Brasil corresponde a 9,1\%. Dos 169.544.443 de cidadãos recenseados, cerca de 14,4 milhões são idosos. De acordo com Kalache et al. (1987), entre 1950 e 2025, a população brasileira crescerá cinco vezes, enquanto a população maior de 60 anos crescerá 15 vezes, posicionando o Brasil em $6^{\circ}$ lugar no mundo, em número absoluto de idosos.

Segundo Litvak (1990), a suscetibilidade a enfermidades crônicas e incapacidades decorre em grande parte do envelhecimento e aumenta a demanda por serviços médicos, sociais e econômicos. Estudos sobre capacidade funcional de idosos, conduzidos no Brasil na década de 1990, encontram altas prevalências de incapacidades para pelo menos uma atividade de vida diária, variando as funções perdidas entre $28,18 \%$ e $61 \%$ (LITVOC; DERNTL, 2002).

Dentre os males crônicos potencialmente incapacitantes, está a doença de Parkinson (DP). De acordo com a Associação Brasil-Parkinson (2005), ela pode afetar qualquer pessoa, independentemente de sexo, raça, cor ou classe social, mas sua incidência e prevalência aumentam com o avanço da idade, e $1 \%$ das pessoas com mais de 65 anos tem a DP.

Embora a causa da DP permaneça desconhecida, parece associar-se com o processo degenerativo de certas estruturas do sistema nervoso central e também com fator genético. Segundo Stoppe e Cabral (1993), a DP é uma doença neurodegenerativa ligada aos gânglios da base.

A DP compromete progressivamente os movimentos da pessoa, com repercussões significativas em seu desempenho funcional. Também pode haver manifestações não motoras como distúrbios de memória e depressão, afetando ainda mais a funcionalidade e a qualidade de vida da pessoa acometida.

\section{Sintomas motores decorrentes da DP e sua repercussão sobre a funcionalidade}

O tremor é o sintoma mais freqüente e o primeiro a ser reconhecido por $70 \%$ das pessoas com DP; é rítmico e relativamente lento, quando comparado com outros tipos de tremor. Ele afeta os dedos ou as mãos e ocorre principalmente quando o membro está em repouso ou segurando um objeto leve, mas pode também se manifestar durante atividades em que se mantém o membro fora do eixo de gravidade e, nesses casos, tem ação mais rápida e se parece mais com o tremor essencial.

No início da doença, o tremor é assimétrico, atinge apenas dedos e pode ser descrito como movimento de "girar-pílula". Com a evolução da DP, passa a ser simétrico, manifestando-se também na cabeça, na mandíbula, nos lábios, no queixo e nos membros inferiores; pode variar durante o dia, ser provocado por estresse emocional e atenuado ou extinto em estado de relaxamento ou sono.

Embora o tremor não seja o sintoma mais incapacitante, freqüentemente afeta a auto-imagem e a auto-estima da pessoa, desencadeando ou agravando sintomas depressivos, inatividade e isolamento social.

A acinesia e a bradicinesia são sintomas bastante comuns na DP e referem-se respectivamente à redução da quantidade de movimentos voluntários e automáticos e à lentidão em sua execução. No estágio inicial da DP, esses sintomas são ainda discretos, embora os movimentos já se tornem mais vagarosos e a pessoa fique mais tempo numa mesma posição. Nesse estágio, à semelhança do tremor, eles afetam, só um dos lados do corpo. Num segundo estágio, pode afetar também o outro, comprometendo mais evidentemente movimentos requeridos para as atividades cotidianas e a marcha. Conforme a doença avança, acentuase a lentidão dos movimentos corporais, podendo culminar com a imobilidade completa.

As repercussões desses sintomas na vida cotidiana da pessoa com DP são indiscutíveis, uma vez que as atividades diárias, que antes eram feitas com rapidez e desembaraço, passam a ser progressivamente mais lentas e a demandar mais esforço. Embora esses sintomas não comprometam a aparência pessoal, são freqüentemente vividos como perda do senso de auto-eficácia e autocontrole, especialmente por aqueles que apresentam sintomas depressivos.

A rigidez muscular é outra característica da doença e decorre do aumento da resistência que os músculos oferecem quando um segmento do corpo é deslocado passivamente. $\mathrm{Na} \mathrm{DP}$, a inibição dos antagonistas não é feita de modo eficaz e, como conseqüência, os músculos ficam mais tensos e contraídos, e a pessoa se sente rígida e com pouca mobilidade. Quando determinado membro é deslocado passivamente, podem-se sentir, superpostos à rigidez, curtos períodos de liberação rítmicos e intermitentes, fenômeno denominado roda denteada. A postura da pessoa com DP também muda, predominando os músculos flexores sobre os extensores, de modo que a cabeça permanece fletida sobre o tronco, este, sobre o abdômen e os membros superiores são mantidos ligeiramente à frente, com os antebraços semi-fletidos na altura do cotovelo. Durante o caminhar, os ombros ficam encolhidos e inclinados para a frente, os 
braços caem paralelos ao corpo e quase não balançam. Os calcanhares arrastam-se no chão, causando um caminhar bastante típico. Quando se senta, a pessoa tende ainda a inclinar a cabeça e encolher os ombros.

As alterações dos reflexos posturais também ocorrem na DP, embora não em estágio inicial. Ocasionam instabilidade postural, mais tarde, disfunção do equilíbrio durante a marcha e em posição ortostática, não permitindo, em estágio mais avançado, que a pessoa se levante ou fique em pé sem assistência.

O fenômeno de freezing, ou bloqueio motor, impede o início do movimento ativo ou o bloqueia; quando associado a alterações dos reflexos posturais, potencializa a ocorrência de quedas.

Além dos sintomas motores, pode haver outras manifestações, requerendo, em alguns casos, abordagem terapêutica/reabilitadora. Dentre eles, citam-se sintomas depressivos, distúrbios cognitivos, distúrbios do sono, dificuldades de fala, sialorréia, dificuldades respiratórias, distúrbios de deglutição, disfunções urinárias, dores musculares e sensações térmicas anormais (http://www. parkinson.med.br. Acesso em 8 nov. 2005).

Merecem destaque os sintomas depressivos, os distúrbios cognitivos e as dificuldades de fala, pela especial consideração que se lhes deve atribuir no processo de reabilitação.

Os sintomas depressivos ocorrem em $40 \%$ a $50 \%$ das pessoas com DP, podendo ser reativos à doença ou compor sua sintomatologia (STOPPE, 1997). Sua intensidade pode variar de quadros leves a graves, em que a depressão é o sintoma mais importante e um dos fatores determinantes de incapacidade. Alterações emocionais como insegurança diante de situações novas também são comuns. As pessoas podem reagir a isso evitando sair de casa e ter contatos sociais. Alguns perdem a motivação e se tornam excessivamente dependentes dos familiares.

Embora a maioria das pessoas com DP não apresente declínio intelectual, podem ocorrer distúrbios cognitivos como dificuldades de concentração e memória para fatos recentes, dificuldades para cálculos e em atividades que requerem orientação espacial. Tais alterações costumam se intensificar com o avanço da doença, especialmente em pessoas idosas. Ainda que esses distúrbios não sejam suficientes para gerar dependência, costumam causar desconforto e preocupação para a pessoa e seus familiares. A demência franca sucede a cerca de $20 \%$ das pessoas acometidas e tem impacto evidente sobre a vida cotidiana (http://www.parkinson.med.br. Acesso em 8 nov. 2005).

Sessenta $(60 \%)$ a $90 \%$ das pessoas com DP desenvolvem dificuldades de fala. A voz se torna mais fraca, seu volume diminui e pode haver certa rouquidão. É comum haver dificuldade na articulação da fala, com conseqüente prejuízo em sua compreensão; essa dificuldade pode se agravar, tornando-se bastante incapacitante nas fases mais avançadas da doença. A palilalia, caracterizada pela aceleração do ritmo da fala com embaralhamento das palavras, também pode ocorrer, perturbando ainda mais a compreensão da fala (http://www.parkinson.med.br. Acesso em 8 nov. 2005).

\section{Recursos tecnológicos: estratégia de promoção de autocuidado, atividades e participação}

O aumento da incidência de doenças crônicas e incapacitantes como a DP "configura-se um desafio para as autoridades sanitárias, em especial no que tange à implantação de novos modelos e métodos para o enfrentamento do problema" (GORDILHO et al., 2000, p. 16).

Como resposta a esse desafio, Litvak (1990) identifica a adoção de medidas específicas para a prevenção de enfermidades da idade avançada e a promoção da saúde.

Na Carta de Ottawa (1986), a promoção da saúde é conceituada como "o processo de capacitação da comunidade para atuar na melhoria de sua qualidade de vida e saúde, incluindo uma maior participação no controle desse processo" (MINISTÉRIO DA SAÚDE, 1996, p. 12).

Segundo Restrepo (1994), a promoção da saúde compreende medidas fundamentais como comunicação social, disseminação de informações, educação para a saúde, promoção de políticas públicas, reorientação dos serviços e desenvolvimento de habilidades pessoais. Com esses mecanismos, trata-se de aumentar o nível de consciência sobre os problemas de saúde prevalentes e a necessidade de se promoverem mudanças comportamentais individuais e sociais, assim como de se obter o compromisso de todos os setores e atores sociais para se levarem a cabo essas mudanças.

Segundo Litvoc e Derntl (2002), na Carta de Ottawa, "coloca-se em relevo o papel das organizações, dos sistemas e das comunidades, bem como a valorização dos comportamentos e das capacidades individuais, como as de autocuidado, na criação de opções e oportunidades para promover a saúde e o desenvolvimento populacional" ( $\mathrm{p}$. 277).

De acordo com Orem (1991), o autocuidado se refere a ações dos indivíduos para manter a vida, a saúde e o bem-estar, que podem ser dirigidas a si próprios ou ao ambiente, atendendo a necessidades pessoais ou de pessoas dependentes.

Ainda segundo a autora, o indivíduo é competente para 
seu autocuidado quando pode discernir fatores que devem ser administrados a fim de regular seu próprio funcionamento e desenvolvimento, decidir o que pode e deve ser feito, planejar os componentes de sua demanda terapêutica de autocuidado (necessidades e métodos e técnicas pelas quais elas possam ser satisfeitas) e, finalmente, tomar medidas para satisfazer a essas necessidades.

Alguns estudos têm se ocupado de identificar práticas de autocuidado adotadas por pessoas com incapacidades e/ou cuidadores para minimizar ou corrigir déficit de autocuidado nas atividades de vida diária. A adoção de RT e de técnicas adaptadas para a realização de atividades se incluem entre essas práticas (NORBURN et al., 1995; ALMEIDA, 1997; GILL et al., 1999; MESSECAR, 2000; GITLIN et al., 2002).

Reconhecem-se as práticas de autocuidado - incluindose a adoção de recursos tecnológicos por pessoas com incapacidades e por integrantes de seu meio familiar e social - como potencialmente facilitadoras não só do desempenho funcional, mas também da autonomia, da participação e da inserção social das pessoas envolvidas.

Encontram-se pontos de intersecção entre a compreensão dos RT como prática de autocuidado e a forma como eles vêm sendo discutidos pela Classificação Internacional de Funcionalidade, Incapacidade e Saúde (CIF). De acordo com a CIF, os RT compõem os fatores ambientais e interagem com os domínios da saúde ou a ela relacionados, designados "funções e estruturas corporais" e "atividades e participação", podendo facilitar a promoção desses domínios e, conseqüentemente, influir nas condições de saúde das pessoas.

As "atividades e participação" de pessoas acometidas pela DP ficam freqüentemente comprometidas, com o avanço da doença, e esse domínio pode ser facilitado pela adoção de RT, por se constituírem recursos potenciais para compensar dificuldades, especialmente de ordem motora e cognitiva.

A adoção de RT inscreve-se como possível estratégia a ser empregada pelo terapeuta ocupacional para a melhora funcional da pessoa com DP. Nesse contexto, seu uso se liga ao exercício de funções específicas e à prática de atividades contextualizadas, o que pode concorrer para o resgate ou a preservação de uma vida cotidiana com significado.

Segundo Heller (1970), o homem se insere na vida cotidiana na medida em que adquire e usa habilidades e, nesse processo, amadurece e se humaniza.

Para resgatar e desenvolver atividades contextualizadas e funções específicas, o terapeuta e a pessoa com DP buscam enfrentar as dificuldades. A adoção de dispositivos é indicada para minimizar a bradicinesia e a acinesia, por seu potencial de facilitar e agilizar as atividades básicas e instrumentais de vida diária. O tremor pode ser atenuado, suas conseqüências, equacionadas e a funcionalidade para atividades diárias pode ser facilitada com o uso de utensílios que tenham algum peso e sejam feitos de materiais inquebráveis. Adaptações no ambiente doméstico e em utensílios visam ainda segurança, conforto, interação e mobilidade, prevenindo a ocorrência de quedas e compensando sintomas como rigidez muscular, instabilidade postural e disfunção do equilíbrio.

Para minimizar dificuldades de memória, sugere-se a adoção de estratégias internas como associação, uso de múltiplos sentidos e categorização de informações. Dispositivos externos à memória como agenda, calendário, lembretes e bilhetes também são indicados, pois poupam a mente de trivialidades favorecendo a organização da vida diária, além de se constituírem, para a maioria das pessoas, recursos familiares, comumente utilizados.

A relação entre usuário e RT tem sido analisada com base na compreensão das dificuldades funcionais e na identificação de dispositivos potencialmente adequados para seu enfrentamento. Embora esse referencial seja importante, não parece suficiente para desvelar a complexidade dessa relação; há que se levar em conta outros referenciais, como o sentido que o usuário dá ao dispositivo, o acesso a ele $\mathrm{e}$ sua permanência na vida cotidiana.

Castiglioni (2003) busca analisar a complexa trama entre pessoa e RT, pondo em questão, a princípio, a afirmação de que o usuário é um indiscutível beneficiário do RT e só lhe caberia adaptar-se a ele. É fundamental compreender a relação ambígua que freqüentemente se estabelece entre usuário e dispositivo, que, de um lado, pode contribuir para a satisfação de necessidades e a inclusão social da pessoa, mas, de outro, pode também representar um fracasso e uma afirmação de sua incapacidade. Assim, o uso desses recursos pode facultar à pessoa liberdade, autonomia e exercício da cidadania, mas é essencial orientar o olhar para a qualidade da inclusão: “(..) os instrumentos em si não definem um paradigma. Seu uso e finalidade dependem dos significados, da intencionalidade e dos pressupostos que referendam o cuidar" (SAWAIA, 1998, p. 15).

A relação entre usuário e RT também pode ser analisada quanto ao acesso ao recurso, muitas vezes dificultado pelo alto custo, decorrente da sofisticação, customização e importação do material de que é comumente feito, assim como quanto à permanência do dispositivo na vida cotidiana da pessoa. Os fabricantes têm buscado equacionar essa questão por meio de conceitos sobre adequação ergonômica, efeitos da atividade gravitacional e microgravidade para maior 
funcionalidade, conforto, segurança e qualidade de vida, especialmente para pessoas com incapacidades. O desenho dos dispositivos evolui para formas progressivamente mais leves, orgânicas, lúdicas e estéticas. Ainda como referenciais para a definição, elaboração e incorporação dos dispositivos à vida cotidiana da pessoa, incluem-se suas expectativas, preferências, idéias e experiências.

Sobre a relação entre usuário com DP e dispositivo, pode-se dizer que o contexto desse usuário é freqüentemente adverso. A prevalência da DP aumenta conforme a idade avança, e, assim, os portadores vivem situações de dupla perda de autonomia e exclusão, tanto por serem idosos quanto por terem a doença. O lugar social que as pessoas com DP comumente ocupam dificulta-lhes o acesso aos dispositivos, sua incorporação ao cotidiano e até mesmo a atribuição de um sentido favorável ao dispositivo, que o associe à promoção de atividades, à participação, à autonomia e à inclusão. Cabe ao terapeuta ocupacional conceber dispositivos que sejam facilitadores de relacionamentos, ensejando o resgate da autonomia e a inclusão. Considera- se que os parâmetros para se obter e avaliar a eficácia dos dispositivos para a pessoa com DP devem ultrapassar sua capacidade de compensar dificuldades motoras e cognitivas e combater sintomas físicos associados à doença, e contemplar especialmente sua contribuição para a satisfação de necessidades, conferindo ou preservando a identidade e a dignidade da pessoa.

\section{CONCLUSÃO}

Muitas profissionais têm se dedicado a conceber e confeccionar recursos tecnológicos, inclusive o terapeuta ocupacional. Para tanto, este analisa a relação entre usuário - inserido num contexto físico e social - e dispositivo, pautando-se na idéia de que essa relação é necessariamente complexa. Os fatores que determinam essa complexidade devem ser constantemente identificados, valorizados e equacionados, para que de fato o dispositivo seja uma ferramenta para a promoção do autocuidado, a consecução de atividades significativas e a participação social.

ALMEIDA, M. H. M., CASTIGLIONI, M. C. Technological resources: strategies of promotion of self-care, activities and the participation of people with Parkinson's disease. Rev. Ter. Ocup. Univ. São Paulo, v.18, n. 3, p. 152-157, set./dez. 2007.

\begin{abstract}
S: The aging of the population is considered a worldwide phenomenon. The susceptibility to chronic diseases and incapacities occur, in great part, due to the effect of aging. Parkinson's disease (PD) is among the potentially incapacitating chronic diseases. It progressively compromises movement and non-motor manifestations may occur, such as memory disturbance and depression. As far as preventive measures for advanced aging diseases and promotion of health, the development of self-help practice is outstanding. The adoption of technological resources (TR) is included in this practice and is a possible strategy to be employed by the occupational therapist to improve functionality. In this context, its utilization is related to the exercise of specific functions and to the practice of contextualized activities. The relation between the user and TR has been habitually analyzed based on the functional difficulties and identification of devices that are potentially adequate for its confrontation. However, it is considered that other referential should be contemplated such as sense attributed to the device by the user, its accessibility and its permanence in daily life. The occupational therapist analyzes the complex relation between user and device, inserted in a physical and social context. In reference to the relation between user with PD and device, it is possible to affirm that the context in which this user is inserted is frequently adverse; this user habitually lives double loss situations of autonomy and exclusion for being elderly as well as for presenting PD. It is up to the occupational therapist to conceive devices that will bring about facilitating function of relationships, favoring the recovery of the patient's autonomy and inclusion.
\end{abstract}

KEY WORDS: Parkinson disease. Self care. Occupational therapy. Health promotion. 
ALMEIDA, M. H. M. et al. Recursos tecnológicos. Rev. Ter. Ocup. Univ. São Paulo, v.18, n. 3, p. 152-157, set./dez. 2007.

\section{REFERÊNCIAS}

ABP - Associação Brasil-Parkinson. O que é Parkinson? [online]. 2005. Disponível em: http://www.parkinson.org.br. Acesso em: 8 nov. 2005.

CIF - Classificação Internacional de Funcionalidade, Incapacidade e Saúde. Centro Brasileiro de Classificação de Doenças. Centro colaborador da Organização Mundial da Saúde para a Família de Classificações Internacionais, org. coordenação da tradução Cássia Maria Buchalla. São Paulo: EDUSP, 2003.

ALMEIDA, M. H. M. CICAc: instrumento para classificação de idosos quanto à capacidade para o autocuidado. São Paulo, 1997. 207 f. Dissertação (Mestrado Saúde Pública) - Faculdade de Saúde Pública da Universidade de São Paulo.

BDPT. Doença de Parkinson? [online]. 2005. Disponível em: http://www.doencadeparkinson.com.br. Acesso em: 8 nov. 2005.

Cabral, A. C. J.; STOPPE, A. J. Alterações psiquiátricas na doença de Parkinson In: FORLENZA, O. V.; CARAMELLI, P. (org.). Neuropsiquiatria geriátrica. São Paulo: Atheneu, 2000. p. 667-677.

CASTIGLIONI, M. C. Entre o exílio e a libertação - uma análise psicossocial da tecnologia assistiva. São Paulo, 2003. 100 f Tese (Doutorado Psicologia Social) - Pontifícia Universidade Católica de São Paulo.

Fundação IBGE. Brasil em números: 2000. Rio de Janeiro, 2000. v.8.

GILL, T. M.; ROBISON, J. T.; WILLIANS, C. S.; TINETTI, M. E. Mismatches between the home environment and physical capabilities among community-living older persons. J. Am. Geriatr. Soc., v. 47, p. 88-92, 1999.

GITLIN, L. N.; WINTER, L.; DENNIS, M. P.; CORCORAN, M.; SCHINFELD, S.; HAUCK, W.W. Strategies used by families to simplify tasks for individuals with alzheimer's disease and related disorders: Psycometric Analysis of Task Management Strategy Index (TMSI). Gerontologist, v. 42. p. 61-9, 2002.

GORDILHO, A.; SERGIO, J.; SILVESTRE, J.; RAMOS, L. R.; FREIRE, M. P. A.; ESPINDOLA, A., et al. Desafios a serem enfrentados no terceiro milênio, pelo setor saúde na atenção integral ao idoso. Rio de janeiro: UnAti, 2000.

HELLER, A. O cotidiano e a história. 2a. ed. São Paulo: Paz e Terra Filosofia, 1970.

KALACHE, A.; Veras, R. P.; Ramos, R.L. O envelhecimento da população mundial: um desafio novo. Rev Saúde Pública, v. 21. p. 200-10, 1987.

LITVAK, J. El envejecimiento de la población: un desafio que va más allá del año 2000. Bol. Of. Sanit. Panam., v. 109. p. 2-5, 1990.

LITVOC, J.; DERNTL, A.M. Capacidade funcional do idoso: significado e aplicações. In: CIANCIARULLO, T. I.; GUALDA, D. M. R.; SILVA, G. T. R.; CUNHA, I. C. K. O. Saúde na família e na comunidade. São Paulo: Robe Editorial, 2002. p.268-319.

MESSECAR, D. C. Caregivers' ability to make environmental modifications. J. Gerontol. Nurs., v. 26. n. 12. p. 32-42, 2000.

MINISTÉRIO DA SAÚDE. Fundação Oswaldo Cruz. Promoção da saúde. Brasília (DF), 1996.

NORBURN, J. E. K. I.; BERNARD, S. L.; KONRAD, T. R.; WOOMERT, A.; DeFRIESE, G. H.; KALSBEEK, W. D., et al. Self-care and assistance from others in coping with functional status limitations among a national sample of older adults. $J$. Gerontol. B Psychol. Sci. Soc. Sci., v. 50. p. 101-9. 1995.

Orem, D. E. Nursing: concepts of practice. 4a. ed. St. Louis: Mosby Year Book, 1991.

PARKINSON online [online]. 2005. Disponível em: http://www. parkinson.med.br. Acesso em: 8 nov. 2005.

RESTREPO, H. E.; PEREZ, E. A. Promoción de la salud de los ancianos. In: PEREZ, E. A.; GALINSK, D.; MARTINEZ, A. R. S.; AYÉNDEZ, M. S. La atención de los ancianos: un desafio para los años noventa. Washington (DC): OMS/OPS, 1994. (POS Publicación Cientifica, 546).

SAWAIA, B. B. Os paradigmas do cuidar. In: $6^{\circ}$ ENFETEC, 1998 , São Paulo. Anais. p. 11-24.

STOPPE, A. J. Característica clínica da depressão em idosos. In: FORLENZA, O. V.; ALMEIDA, O. P. (Org.). Depressão e demência no idoso. São Paulo: Lemos Editorial, 1997. p. 47-68. 